\title{
Gender differences in vaccine therapy: where are we in Covid-19 pandemic?
}

\author{
Tiziana Ciarambino1, Elena Barbagelata ${ }^{2}$, Graziamaria Corbi ${ }^{3}$, Immacolata Ambrosino ${ }^{4}$, Cecilia Politi ${ }^{5}$, \\ Franco Lavalle ${ }^{6}$, Anna Ruggieri ${ }^{7}$, Anna Maria Moretti ${ }^{8}$
}

${ }^{1}$ Internal Medicine Department, Marcianise Hospital, ASL Caserta; ${ }^{2}$ Department of Internal Medicine, Lavagna Hospital, Genova; ${ }^{3}$ Department of Medicine and Health Sciences, University of Molise; ${ }^{4}$ Local Healthcare Unit of Bari, Health District 10, Post-acute Care Triggiano, Bari; ${ }^{5}$ Head of Gender Medicine Area F.A.D.O.I., Internal Medicine Department, Isernia; ${ }^{6}$ Vicepresidente OMCEO Bari; ${ }^{7}$ Center for Gender Specific Medicine, Istituto Superiore Sanità, Rome; ${ }^{8}$ President of GISeG, Italian Group for Health and Gender, Bari, Italy

\begin{abstract}
Vaccination is one of the greatest achievements of public health. Vaccination programs have contributed to the decline in mortality and morbidity of various infectious diseases. This review aims to investigate the impact of sex/gender on the vaccine acceptance, responses, and outcomes. The studies were identified by using PubMed, until $30^{\text {th }}$ June 2020 . The search was performed by using the following keywords: SARS-CoV-2, Covid-19, gender, sex, vaccine, adverse reaction. Clinical trials, retrospective and prospective studies were included. Studies written in languages other than English were excluded. Three authors (TC, EB and IA) reviewed all study abstracts. Studies were included if gender differences in response to vaccination trials were reported. All
\end{abstract}

Correspondence: Tiziana Ciarambino, Internal Medicine Department, Marcianise Hospital, ASL Caserta, Italy.

E-mail: tiziana.ciarambino@gmail.com

Authors' contributions: TC study conception and design, revision of all clinical trials, manuscript drafting. TC, IA, EB, GMC revision of all clinical trials, critical revision. AR, CP, FL, AMM study conception and design, critical revision. All authors read and approved the final version of the manuscript.

Conflict of interest: The authors declare that they have no conflicts of interest related to the subject matter or materials discussed in this article.

Availability of data and materials: All data generated or analyzed during this study are included in this published article.

Key words: Gender and/or sex differences; vaccine; adverse reaction; Covid-19; SARS-CoV-2.

Received for publication: 7 November 2020.

Accepted for publication: 24 March 2021.

${ }^{\circ}$ Copyright: the Author(s), 2021

Licensee PAGEPress, Italy

Monaldi Archives for Chest Disease 2021; 91:1669

doi: 10.4081/monaldi.2021.1669

This article is distributed under the terms of the Creative Commons Attribution Noncommercial License (by-nc 4.0) which permits any noncommercial use, distribution, and reproduction in any medium, provided the original author(s) and source are credited. selected studies were qualitatively analyzed. Innate recognition and response to viruses, as well as, adaptive immune responses during viral infections, differ between females and males. Unfortunately, a majority of vaccine trials have focused on healthy people, with ages between 18 to 65 years, excluding the elderly, pregnant women, post-menopausal female and children. In conclusion, it is apparent that the design of vaccines and vaccine strategies should be sex-specific, to reduce adverse reactions in females and increase immunogenicity in males. It should be mandatory to examine sex-related variables in pre-clinical and clinical vaccine trials, such as their crucial role for successful prevention of pandemic Covid-19.

\section{Introduction}

Vaccination is one of the greatest achievements of public health. Vaccination programs have contributed to the decline in mortality and morbidity of various infectious diseases [1]. To be successful in reducing the prevalence and incidence of vaccinepreventable diseases (VPD), vaccination programs rely on a high uptake level. In addition to direct protection for vaccinated individuals, high vaccination coverage rates induce indirect protection for the overall community, or herd immunity, by slowing transmission of VPD, thereby decreasing the risk of infection among those who remain susceptible in the community [2]. However, these national estimates may hide clusters of under-vaccinated individuals [3]. It is estimated that less than $5-10 \%$ of individuals have strong anti-vaccination convictions [4]. However, a more significant proportion could be categorized as being hesitant regarding vaccination [5]. In particular, sex and gender-related factors, impact vaccine acceptance, responses, and outcomes. Sex (i.e., the biological differences between males and females) and gender (i.e., cultural norms associated with being male or female) impact acceptance of, responses to, and the outcome of vaccination [6]. Female individuals are often less likely to accept vaccines, but once vaccinated, develop higher and longer-lasting protective antibody responses, as compared to males [6]. However, they undergo adverse events more often and intense than male vaccines [6]. The mechanisms underlying sex differences in response to vaccination include sex hormone modulation of the immune system as well as genetic and epigenetic regulation. It has been reported that the immune response to the monovalent 2009 H1N1 vaccine, was partly due to reproductive senescence in females, in which higher circulating estradiol concentrations are associated 
with greater antibody responses to the vaccine [7]. Although there are distinct effects of sex steroid hormones on immune responses, including responses to vaccines, the lack of age-related changes in the sex differential effects of vaccines suggests that genetic or other factors are likely to be involved. This review aims to investigate the impact of sex/gender on the vaccine acceptance, responses, and outcomes.

\section{Methods}

The studies were identified by using the PubMed database, published until $30^{\text {th }}$ June 2020 . The search was performed by using the following keywords: SARS-CoV-2, Covid-19, gender, sex, vaccine, adverse reaction. Clinical trials, retrospective, and prospective studies were included. Three authors (TC, EB and IA) reviewed all study abstracts. Studies were included if gender differences in response to the vaccine were reported. Studies written in languages other than English were excluded. All selected studies were qualitatively analyzed.

\section{Results}

\section{Vaccine and gender}

In our previous review, we underlined that the reduced susceptibility of females to viral infections could be attributed to the protection from the $\mathrm{X}$ chromosome and sex hormones, which play an essential role in innate and adaptive immunity [8].

In particular, it has been reported that the innate immune response to viruses, as well as adaptive immune responses during viral infections, differ between females and males [7]. Adult females develop higher antibody responses to vaccines than males. After either childhood or adult vaccination against influenza, yellow fever, rubella, measles, mumps, hepatitis A and B, herpes simplex 2, rabies, dengue viruses, protective antibody responses can be twice as high in females compared to males of all ages [6]

In this regard, several studies have illustrated that females, generally have greater inflammatory and humoral immune responses, than males, during viral infections [7] ( $\square$ Supplementary Figure 1). These responses contribute to better clearance of viruses, including SARS-CoV-2, in female subjects. To this regard, in Covid-19 infection, cytokine response is higher in acute respiratory distress syndrome (ARDS) male patients. A study of 331 Chinese patients, with confirmed SARS-CoV-2 infection, reported the anti-SARS-CoV-2 immunoglobulin $\mathrm{G}$ ( $\mathrm{IgG}$ ) responses were related to the severity of the disease. The sex distribution of recovering cases was $36 \%$ and $65 \%$ for men and women, respectively [9]. Interestingly, the anti-SARSCoV-2 IgG titers were similar between sexes in patients with mild Covid-19 disease. In patients with severe disease, the female subjects exhibited a higher antibody response, than men, with the production of antibodies at earlier phases of the disease. These results were also consistent with other studies describing higher antibody levels (including more functional antibodies) in adult females compared to adult males [7]. Some of these differences can be linked to hormonal differences, such as estrogen and testosterone levels [10]. The more intense immune responses in women predispose female subjects to autoimmune diseases, and to experience more adverse reactions to the vaccine [11]. It has been reported that testosterone reduces vaccination response [12]. In a randomized, prospective, single-blind study on humoral immune responses in an inactivated influenza vac- cine (TIV), Beyer et al. reported that healthy women (18-64 years of age) generate a more robust protective antibody response. The response of female to a half dose of the influenza vaccine, was equivalent to the antibody response of men to the full dose [13], thus posing the basis for a sex-personalized vaccine dosage.

One possible explanation of the more intense, and sometimes, longer-lasting immune responses in women is because females have two X chromosomes, while men only one. Since the X chromosome is enriched by genes encoding immune factors (such as Toll-like Receptor-7 [TLR7], interleukins, and chemokines) as regulatory molecules like microRNAs, there is an overexpression of immunerelated molecules in female subjects compared to males.

Age is another factor that induces the modification of immune response and it should also not be forgotten. In this regard, with aging, there is a progressive functional decline in the immune system [14]. One of the most well-characterized attributes of an aging immune system is an aberrant chronic low-grade pro-inflammatory state, which may occur to a greater extent in females than in males [15]. Moreover, Wang et al. evidenced that, in older women, influenza vaccination has been associated with lower hospitalization and mortality rates compared to men, suggesting that females maintain higher titers of hemagglutination inhibition (HAI) assay, or respond more effectively to vaccination, thereby experiencing better protection in comparison to men [16].

\section{Covid-19 vaccine and gender}

There is a strong consensus globally that a Covid-19 vaccine is likely the most effective approach to control the Covid-19 pandemic. Coronavirus vaccines, in animal models that mimic human disease, have been immunogenic but generally not shown to effectively prevent the disorder [17]. Previous use of coronavirus vaccines (SARS-CoV and MERS-CoV) in some animal models raised safety concerns regarding Th2 mediated immunopathology [18]. As of $1^{\text {st }}$ June 2020, there are currently 124 candidate vaccines that are under development for prevention of Covid-19 disease, listed by the World Health Organization (WHO) [19] of which 10 candidate vaccines, specifically designed for the prevention of Covid-19 have entered phase 1 , combined phase $1 / 2$ or phase 2 human clinical trials in adults. Most of these trials are enrolling healthy adults (from age 18 to 55 years) only, with the upper age limit of inclusion ranging from 50 to 60 years. Two trials are enrolling young participants, one from aged $\geq 3$ years and the other $\geq 6$ years with no upper age limit in both. One combined phase I/II trial, includes older adults (up to age 85 years), while another early phase I, been extended in May 2020 to include also older adults (to age 99 years) [20]. Unfortunately, a majority of vaccine trials have focused on healthy people, between the ages of 18-65 years, excluding the elderly, pregnant women, post-menopausal females and children. In this regard, it is suggested that post-menopausal female will need a tailored vaccine dose, to contrast their susceptibility to infectious diseases once their estrogen levels decline [21]. Therefore, given the disproportionate mortality rate by gender in people over the age of 60 , the elderly and gender need to be considered in vaccine trials to ensure safety, immunogenicity and efficacy. Vaccine efficacy in under-represented (female subjects for example) and vulnerable populations (older population for example) also remains an issue. An important point to be considered comes from the recently disclosed preliminary results of two ongoing Covid-19 vaccine clinical trials [22] reporting that the population under study included male and female subjects. However, the published results about safety and immunogenicity are not stratified by sex, thus probably underestimating the adverse effects in women and the sex-specific immunogenic response. 


\section{Discussion}

Males and females are biologically different, and this fact likely contributes to sex-specific vaccine outcomes. Among children, adults and older adults, females develop higher antibody responses and experience more adverse reactions to vaccines than males. In particular, the elderly may represent a specific cluster of high-risk patients for developing Covid-19 with rapidly progressive clinical deterioration. Indeed, in older individuals, immune-senescence and comorbid disorders are more likely to promote viral-induced cytokine storm resulting in life-threatening respiratory failure and multisystemic involvement [23]. Although there are distinct effects of sex steroid hormones on immune responses, including responses to vaccines, the lack of age-related changes in the sex differential effects of vaccines suggests that genetic or other factors are likely to be involved. In this global health emergency, the vaccine is needed, considering the role of sex in vaccine response and adverse reactions. It is crucial that gender is considered in clinical trials for Covid-19 vaccines-development to include the analyses of efficacy, vaccine response and adverse reactions. In our review, we analyzed clinical trials, which evaluated immune responses by gender. In all the analyzed studies, we did not find data disaggregated by sex and age, related to effectiveness, adverse reactions and vaccine response. Current pandemic stressed the importance to allowing a large access to highly attractive investigational drugs in international programs and to authorize a more rapid extension of the approved drugs indications as performed in clinical settings other than Covid-19 [24].

As reported for other treatments, each patient is unique, so we can't think to gain the same response for everyone. For this reason, it is crucial to collect and analyze sex-disaggregated data, to test and identify, specific prophylactic and treatment measures. It is urgently needed to obtain a gender-specific preventive intervention by vaccination based on personalized vaccination. In conclusion, sex/gender variables should be considered in preclinical and clinical vaccine trials as a crucial role in the successful prevention of the Covid-19 pandemic.

\section{References}

1. Centers for Disease Control and Prevention (CDC). Ten great public health achievements - United States, 1900-1999. MMWR Morb Mortal Wkly Rep 1999;48:241-3.

2. Fine P, Eames K, Heymann DL. "Herd immunity": a rough guide. Clin Infect Dis 2011;52:911-6.

3. Omer SB, Salmon DA, Orenstein WA, et al. Vaccine refusal, mandatory immunization, and the risks of vaccine-preventable diseases. N Engl J Med 2009;360:1981-8.

4. Leask J. Target the fence-sitters. Nature 2011;473:443-5.

5. Leask J, Kinnersley P, Jackson C, et al. Communicating with parents about vaccination: a framework for health professionals. BMC Pediatr 2012;12:154.

6. Klein SL, Jedlicka A, Pekosz A. The Xs and Y of immune responses to viral vaccines. Lancet Infect Dis 2010;10:338-49.

7. Potluri T, Fink AL, Sylvia KE, et al. Age associated changes in the impact of sex steroids on influenza vaccine responses in males and females. NPJ Vaccines 2019;4:29.

8. Ambrosino I, Barbagelata E, Ortona E, et al. Gender differences in patients with Covid-19: a narrative review. Monaldi Arch Chest Dis 2020;90:1389.

9. Zeng F, Dai C, Cai P, et al. A comparison study of SARS-CoV2 IgG antibody between male and female Covid-19 patients: a possible reason underlying different outcome between gender. J Med Virol 2020;92:2050-4.

10. Ruggieri A, Anticoli S, D'Ambrosio A, et al. The influence of sex and gender on immunity, infection and vaccination. Ann Ist Super Sanita 2016;52:198-204.

11. Fischinger S, Boudreau CM, Butler AL, et al. Sex differences in vaccine-induced humoral immunity. Semin Immunopathol 2019;41:239-49.

12. Trigunaite A. Suppressive effects of androgens on the immune system. Cell Immunol 2015;294:87-94.

13. Beyer WE, Palache AM, Kerstens R, Masurel N. Gender differences in local and systemic reactions to inactivated influenza vaccine, established by a meta-analysis of fourteen independent studies. Eur J Clin Microbiol Infect Dis 1996;15:65-70.

14. Castelo-Branco C, Soveral I. The immune system and aging: a review. Gynecol Endocrinol 2014;30:16-22.

15. Fink AL, Klein SL. Sex and gender impact immune responses to vaccines among the elderly. Physiology (Bethesda) 2015;30:408-16.

16. Wang CS, Wang ST, Chou P. Efficacy and cost-effectiveness of influenza vaccination of the elderly in a densely populated and unvaccinated community. Vaccine 2002;20:2494-9.

17. Roper RL, Rehm KE. SARS vaccines: where are we? Expert Rev Vaccines 2009;8:887-98.

18. Graham RL, Donaldson EF, Baric RS. A decade after SARS: strategies for controlling emerging coronaviruses. Nat Rev Microbiol 2013;11:836-48.

19. World Health Organization. DRAFT landscape of Covid-19 candidate vaccines. World Health Organization; 2020. Available from: https:/www.who.int/docs/default-source/afuturefor-children/novel-coronavirus_landscape_covid19.pdf?sfvrsn=4d8bd201_1\&download=true

20. Clinical Trials.gov [Internet]. Bethesda (MD): National Library of Medicine (US). Identifier: NCT04357028. Measles vaccine in HCW (MV-COVID19). 2020 April 22. Available from: https://clinicaltrials.gov/ct2/show/NCT04357028

21. Cutolo M, Smith V, Paolino S. Understanding immune effects of oestroges to explain the reduced morbidity and mortality in female versus male Covid-19 patients: comparison with autoimmunity and vaccination Clin Exp Rheumatol 2020;38:383-6.

22. Jackson LA, Anderson EJ, Rouphael NG, et al. An mRNA Vaccine against SARS-CoV-2 - Preliminary report. N Engl J Med 2020;383:1920-31.

23. Perrotta F, Corbi G, Mazzeo G, et al. Covid-19 and the elderly: insights into pathogenesis and clinical decision-making. Aging Clin Exp Res 2020;32:1599-608.

24. Pagliano P, Scarpati G, Sellitto C, et al. Experimental pharmacotherapy for Covid-19: The latest advances. J Exp Pharmacol 2021;13:1-13. 